\title{
Investigation of Resonance and Ferroresonance Overvoltages due to Cable- Transformer Interactions
}

\author{
Alyaa A. Nassar ${ }^{1}$, Abdel-Maksoud I. Taalab ${ }^{2}$, Mohamed A. Izzularab ${ }^{2}$, and \\ Nagy I. Elkalashy ${ }^{2 *}$ \\ ${ }^{1}$ Ph.D. Candidate, Electrical Engineering Department, Faculty of Engineering, Menoufia \\ University, Shebin Elkom, 32511, Egypt. \\ ${ }^{2}$ Electrical Engineering Department, Faculty of Engineering, Menoufia University, Shebin Elkom, \\ 32511, Egypt. \\ (Corresponding author: nagy.elkalashy@sh-eng.menofia.edu.eg)
}

\begin{abstract}
In this paper, the resonance and ferroresonance phenomena are deeply investigated considering two different cable-transformer power systems. The internal transformer resonance overvoltage case is initiated due to a single-phase earth fault, while the studied ferroresonance case is due to a single-phase switching in the source feeding an unloaded transformer via a power cable. The resulted secondary overvoltage and the core flux signatures are used as a classifier, where the transient features are extracted using three algorithms. Although the voltage frequency spectrum and phase-plane diagrams have been previously utilized for the ferroresonance mode evaluation, they are exploited in this paper for the comparison study and accordingly for the detection of resonance and ferroresonance phenomena. Salient features found in the transients extracted using the well-known DWT are used as inputs of the third algorithm. A new detection approach is attained based on the DWT-based transient feature extraction of resonance and ferroresonance overvoltages.
\end{abstract}

Keywords: Resonance and ferroresonance overvoltages; cable-transformer interaction; phase-plane diagram; DWT.

\begin{abstract}
1. Introduction
Power transformers may suffer from insulation failure due to their internal resonance or ferroresonance overvoltages. Therefore, each of these two phenomena is to be identified and distinguished.

The internal resonance of the power transformers is triggered by transient surges in the power system that may be due to faults or capacitor switching [1-2]. These surges at the transformer primary side are below its overvoltage protection level, and therefore, they are not damped. Accordingly, they transmit through the transformer windings providing overvoltages at the secondary side. When the transient surge waveform contains frequency matched the resonance frequency of the transformer windings, the internal transformer resonance is happened and provides transient overvoltages. However, it can result in insulation breakdown for transformer winding due to the resulted highfrequency overvoltages at the secondary side [3-8]. Overvoltage protections such as surge arrestor can be
\end{abstract}

installed at the secondary side of the transformer in order to mitigate such induced overvoltages .

The ferroresonance is a special case of the transformer internal resonance that characterized by the existence of several operating steady-state responses. Accordingly, it may exhibit different modes of operation [9-15]. The ferroresonance is initiated due to a single-pole switching or fuse blowing of the source supplying unloaded transformer through an underground power cable. This action produces a series resonance of low frequencies between the magnetizing reactance of the transformer and the system capacitances [11-17]. Ferroresonance causes transformer overvoltage or undervoltage for a long period with high-sustained levels of distortion in the voltage and current waveforms. It produces equipment damage, insulation failure, and apparent mal-operation of protective devices [9-10]. Detection algorithms for these two phenomena either internal resonance or ferroresonance should be proposed for enhancing the condition monitoring of the cable-transformer 
systems and for providing a protection action if needed.

In [18], DWT and ANN were used for the discrimination between temporary and permanent overvoltages across the power transformer due to different events. The studied events include ferroresonance and capacitor switching, where the three-phase secondary currents are used as a classifier. In [19], DWT and ANN were also used to distinguish ferroresonance from the other transients. In [20], DWT is exploited for detecting ferroresonance and identifying its frequency, and consequently, its mode is identified. In [21], DWT was used for distinguishing between isolated capacitor switching and back-to-back capacitor switching. In [22-23], DWT and fuzzy interface system was used to differentiate between transformer magnetizing inrush current and internal fault current. However, these algorithms depended on the artificial intelligent technique to find suitable features for the discrimination point of view, and they could not present salient features utilized for the discrimination. This paper focuses on the investigation of overvoltages for cable-transformer power systems and therefore distinguishing between the internal resonance and ferroresonance. The studied resonance test case is due to a fault occurrence, while the ferroresonance test case is due to a single-phase switching. These two test case studies are considered for two different power transformers, one is the conventional two-winding three-phase power transformer and the second one is the three-winding three-phase autotransformer. The simulation results are carried out to attain internal resonance and ferroresonance overvoltage case studies using the alternative transient program-electromagnetic transient program (ATP-EMTP). The feature extractions for these overvoltages are exploited in order to present a novel distinguishing technique of each disturbance using effective methods. These methods are based on digital signal processing such as fast Fourier transform (FFT), phase-plane diagram, and discrete wavelet transform (DWT). The integration between these algorithms provides better distinguishing performance.

\section{Simulated Case Studies}

The study is carried out for two unloaded power transformers. The first transformer is a 155 MVA, $132 / 15 \mathrm{kV}, \mathrm{Y} / \Delta$, three-phase, two-winding traditional power transformer. The other one is a $250 \mathrm{MVA}$, 400/132/18 kV, Y/Y/ $\Delta$, three-phase, three-winding autotransformer. Each of these two transformers is fed through a single core XLPE underground power cable as shown in Figure (1). The cable rating is selected considering the primary side rating. This figure shows two different disturbance sources for attaining overvoltage measurements of resonance and ferroresonance phenomena.

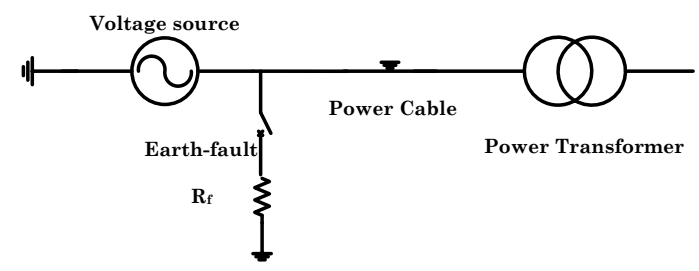

a) Internal transformer resonance due to earth fault.

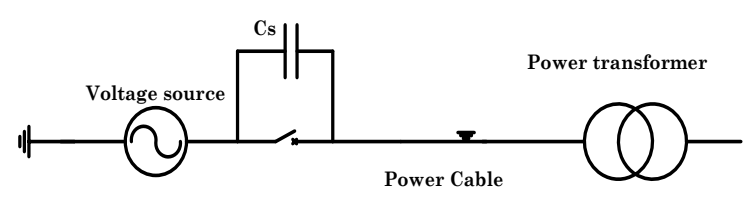

b) Ferroresonance due to primary circuit switching.

Figure 1- Studied power systems.

Using the ATP program, the power transformers are modeled using BCTRAN subroutine. The BCTRAN drives a linear representation of the power transformer, using excitation and short circuit test data that are summarized in the Appendix. As the BCTRAN only produces a linear representation, three nonlinear Type-96 hysteresis inductors are connected at the delta side of each transformer [24]. The considered hysteresis characteristics for each transformer are shown in the Appendix. The highfrequency representation is considered by adding the winding capacitances as external components as reported and given in the ATPDraw manual [24], in which the two-winding transformer has winding stray capacitances considered $0.01 \mu \mathrm{F}, 0.005 \mu \mathrm{F}$, and 0.01 $\mu \mathrm{F}$ for primary to secondary, primary to ground, and secondary to ground; respectively. While the winding capacitances of the three-winding autotransformer are considered $1 \mu \mathrm{F}, 1 \mu \mathrm{F}$ and $0.01 \mu \mathrm{F}$ for primary windings to ground, primary to tertiary winding, and tertiary windings to ground; respectively. The frequency-based JMarti model is used for the underground power cable representation, which gives a frequency-dependent model with a constant transformation matrix based on the cable geometry and its material constants [24]. The simulation is done using the ATP-EMTP program and preprocessed using ATPDraw. The cable data are defined in the Appendix.

\subsection{Resonance Case Study}

Internal transformer resonance overvoltages are commonly due to capacitor switching or faults. It was found that each of these two disturbances has the same resonance overvoltages as both of them provide 
a sudden chopping in the voltage waveform producing traveling surges over the network [25]. The overvoltages due to capacitor switching can be mitigated by controlling the switching inception angle and avoiding the critical cable length at which the maximum overvoltage occurs. Such techniques do not mitigate the overvoltages due to faults as the faults randomly occur at any inception angle and at different fault distances over the cable. Therefore, this paper focuses on the fault based-resonance overvoltages.

The system shown in Figure (1.a) is used to simulate the resonance occurrence during single-phase earth fault for the two studied transformers. The corresponding resonance transients at the secondary side of each power transformer are shown in Figure (2). For the two-winding power transformer as in Figure (2.a), the worst condition for the earth fault is considered when the fault inception angle is $90^{\circ}$ that is corresponding to the instant $0.08833 \mathrm{~s}$. For more declaration, the peak instant is at $0.05 \mathrm{~s}$ if the simulation time started at the voltage zero crossings. However, the voltage peak instant is first estimated from the simulation waveform and it is found at $0.08833 \mathrm{~s}$ that is considered as the fault inception instant to provide the inception angle $90^{\circ}$. The cable length is $900 \mathrm{~m}$ at which its resonance frequency is close to the transformer resonance frequency. At this critical length, the resonance overvoltage has consequently found the maximum value, and the fault is considered solidly to produce the highest transient amplitudes. At these conditions when phase-c to earth fault occurs, a transient voltage oscillation is generated over the power cable. However, the amplitude of this transient is less than the primary circuit peak voltage. Accordingly, the overvoltage protection at the transformer primary side could not limit or diminish these transients that therefore trigger internal transformer resonance causing transient overvoltages at the secondary side as depicted in Figure (2.a).

For the three-winding autotransformer as depicted in Figure (2.b), phase-c to earth fault occurs at $0.0933 \mathrm{~s}$, which corresponds to the $90^{\circ}$ fault inception angle. The underground feeding cable length is considered $100 \mathrm{~m}$, and the fault is considered solidly. Figure (2.b) shows the secondary side overvoltage that occurs due to the internal transformer resonance. These voltage waveforms for the two studied transformers are analyzed in the following section for evaluating the proposed algorithms.

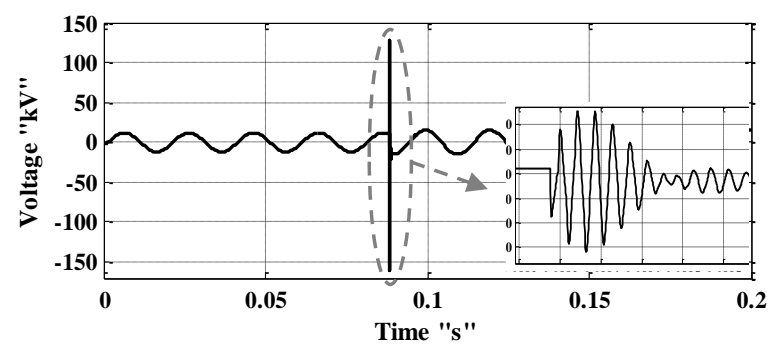

a) Two-winding conventional transformer.

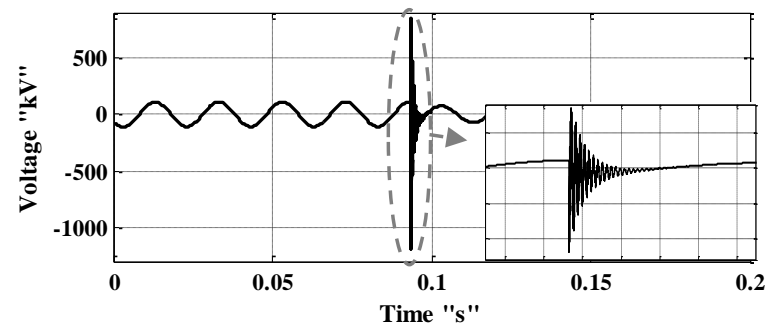

b) Three-winding autotransformer.

Figure 2- Phase-c secondary side voltage waveform due to resonance.

\subsection{Ferroresonance Case Study}

Ferroresonance phenomenon is studied using the simulated power system shown in Figure (1.b). As considered in the resonance test cases, the same cable lengths are taken $900 \mathrm{~m}$ and $100 \mathrm{~m}$ for ferroresonance evaluations of the two-winding and three-winding power transformers, respectively. The circuit breaker is used to initiate ferroresonance due to single-phase switching its grading capacitance is considered $8 \mathrm{nF}$ and $2 \mathrm{nF}$ for cases of the twowinding and three-winding transformers, respectively. Ferroresonance occurs as a result of switching only the phase-c circuit breaker at the instant $0.03 \mathrm{~s}$ for the simulation time. Figure (3) shows the measured secondary voltage under the ferroresonance. The voltage waveforms for the two transformers are distorted with an increase in their magnitude after the switching.

The ferroresonance transients are influenced by the power cable length due to changing its equivalent capacitances. These capacitance changes affect the ferroresonance operating points on the nonlinear magnetic characteristics. Note that the cable lengths in both studied cases that yield overvoltages either due to resonance or ferroresonance can be found in the field in front of the transformer substation. Also, the overhead transmission lines produce the same effect of underground cables, however, for long line lengths. Therefore, discriminating and detecting these overvoltages should be considered to either diagnose the event or evaluate toward acting as soon as possible to save transformers. 


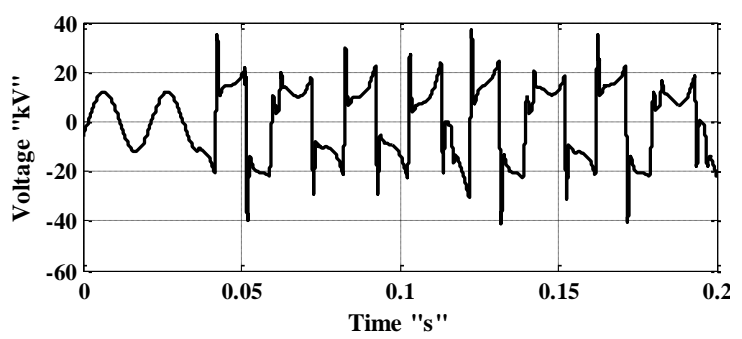

a) Two-winding conventional transformer.

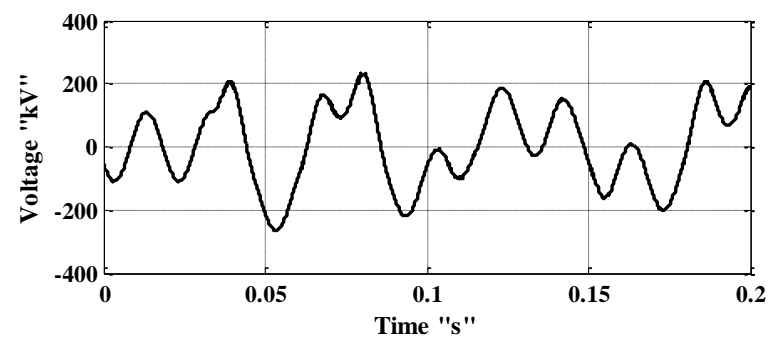

b) Three-winding autotransformer.

Figure 3- Phase-c secondary side voltage waveform due to ferroresonance.

\section{Investigation and Distinguishing Algorithms}

The resonance feature is transient overvoltages at the transformer secondary side that are quickly diminished. Its detection should be recognized with the transient records associated with fault or switching events. The resonance distinguishing approach can be used as an input to the network condition monitoring. An alarm is initiated to call the enhancement of overvoltage protection. However, the ferroresonance feature is highly stressed on the magnetic core of the transformer that its period is extremely long. The detection should be accomplished to isolate the power transformer for limiting the ferroresonance overvoltages or to add devices mitigating the ferroresonance. Even if the transformer damage happened, their discrimination is still important to diagnose the event and add a suitable mitigation method.

As the internal transformer resonance phenomenon is characterized by high-frequency overvoltages during the transient period [7], the core flux decreases where the voltage is proportional to the frequency and flux (E $\propto$ f. $\phi$ ). However, the ferroresonance is characterized by the saturated iron core [9], high magnetizing currents, sustained overvoltages, and distortion in the waveforms. The ferroresonance is considered a low-frequency overvoltage. From the features of each phenomenon, the frequency analysis and core flux are exploited as distinguishing factors. Furthermore, the DWT is found as a useful tool to extract the power system transients, and so it is used with the current two phenomena. These algorithms are discussed as follows.

\subsection{Investigation Based on Frequency Analysis}

As shown in Figures (2) and (3), they are the corresponding overvoltage waveforms due to internal resonance and ferroresonance for both transformers, respectively. Figure (4) shows the frequency spectrum analysis in the case of the two-winding power transformer for both studied cases. The frequency spectrum shown in Figure (4.a) explains high frequencies with significant magnitudes in case of internal transformer resonance, and the high resonance frequencies are about $49,141,238,328, \ldots$ $\mathrm{kHz}$ during the transient period. In the case of ferroresonance as zoomed and depicted in Figure (4.b), the obvious frequencies are the low frequencies of subharmonics and harmonics of the fundamental frequency, in which they are at 15.6, 30.5, 45.7, 53.4, $61,76.3,91.5,114, \ldots \mathrm{Hz}$. By evaluating the threewinding autotransformer, the frequency spectrum analysis for both resonance and ferroresonance cases are shown in Figure (5). In the case of the resonance event, there are high resonance frequencies such as 4.25 and $42.7 \mathrm{kHz}$ as shown in Figure (5.a). While in the case of ferroresonance shown Figure (5.b), the low resonance frequencies are found at 18.3, 30.52, 48.83, 73.24, 85.43, 97.6, ... Hz.

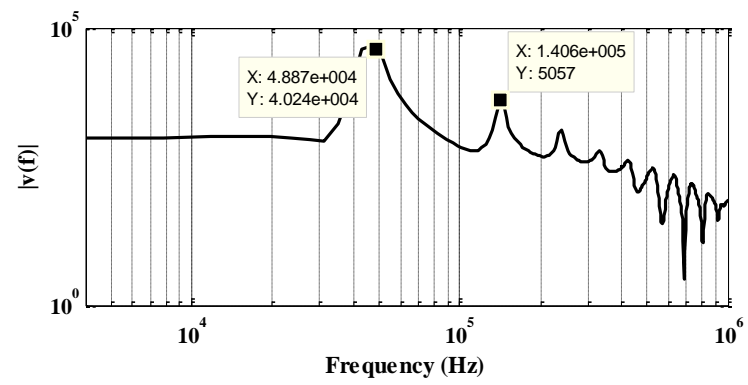

a) High resonance frequencies due to the transformer internal resonance.

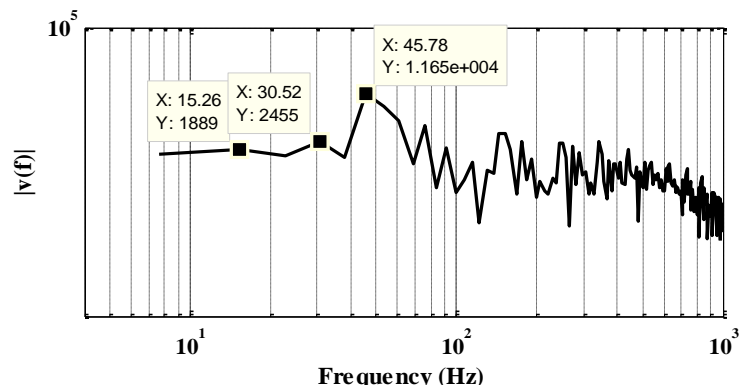

b) Resonance frequencies due to the ferroresonance.

Figure 4- Frequency spectrum analysis for twowinding transformer. 


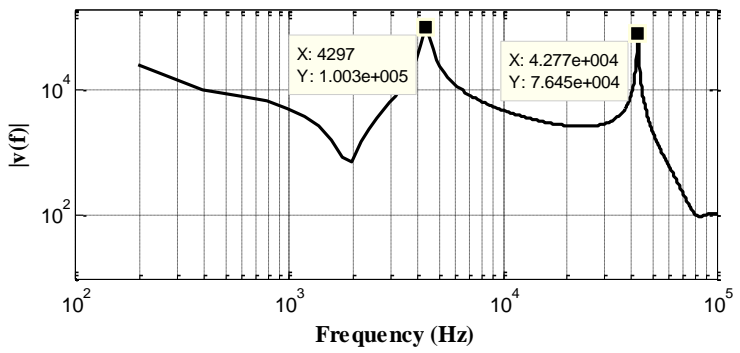

a) High resonance frequencies due to the transformer internal resonance.

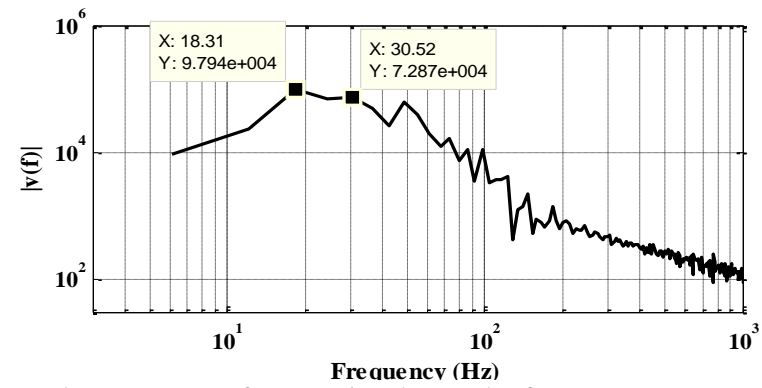

b) Resonance frequencies due to the ferroresonance.

Figure 5- Frequency spectrum for there-winding autotransformer.

From the results, the FFT frequency spectrum is compared to distinguish between these two phenomena. Internal transformer resonance is distinguished with the existence of high frequencies in terms of $\mathrm{kHz}$, while the low frequencies support to distinguish the ferroresonance. The features extracted using FFT are exploited to find a detection procedure as follows:

1. Evaluate the FFT as in Figures (4) and (5).

2. If the high-frequency resonances are detected, then this can be an internal resonance. For the time domain, the overvoltages are diminished, quickly.

3. When a sustained overvoltage is detected including subharmonics, inter-harmonics, and harmonics, this case can be a ferroresonance. In the time domain, there are distorted overvoltages and overcurrents for a long period.

The features extracted using FFT and time-domain observations of internal resonance and ferroresonance cases can be conflicted with the other power system disturbances. Therefore, the detection security is enhanced by the following studied approaches.

\subsection{Investigation Based on Phase-Plane Diagram}

The second algorithm used for distinguishing between the resonance and ferroresonance is based on the phase-plane diagram, where the core flux is plotted against voltage for the same phase. The phase-plane diagrams in the case of internal transformer resonance for both studied transformers are shown in Figure (6). It shows three operating states that are normal operation (blue color), transient period (black), and steady-state period after the transient period (red). The flux band sharply decreases with a voltage increase under the internal transformer resonance during the transient period for both transformers. Figure (7) shows the phase-plane diagram in case of ferroresonance for both studied transformers, which represents several operating loops beside the normal operating loop. Also, the flux band for each of these loops during ferroresonance is higher than the flux band during normal operation that confirms saturation due to ferroresonance.

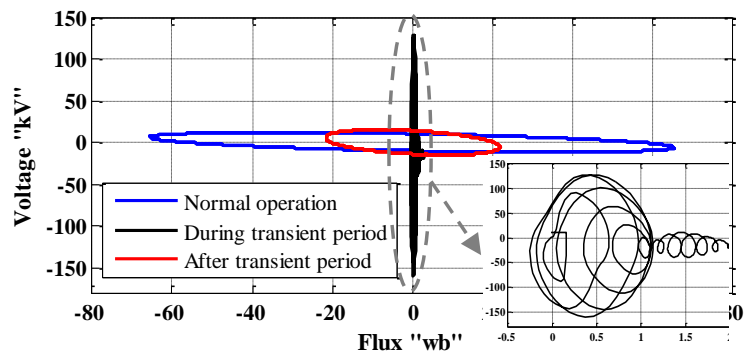

a) Two-winding transformer.

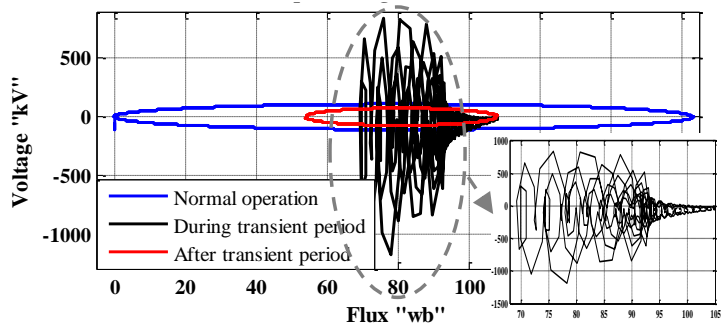

b) Three-winding autotransformer.

Figure 6- Phase-plane diagram due to resonance.

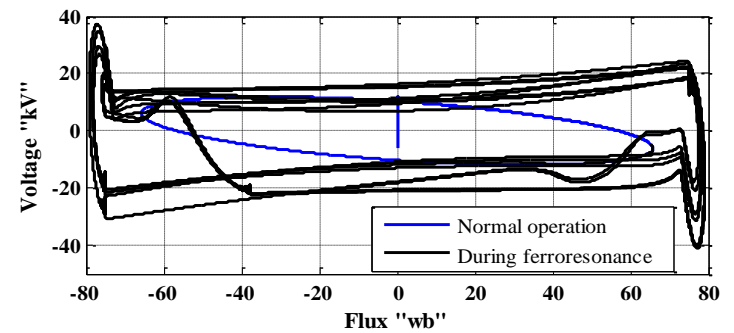

a) Two-winding transformer.

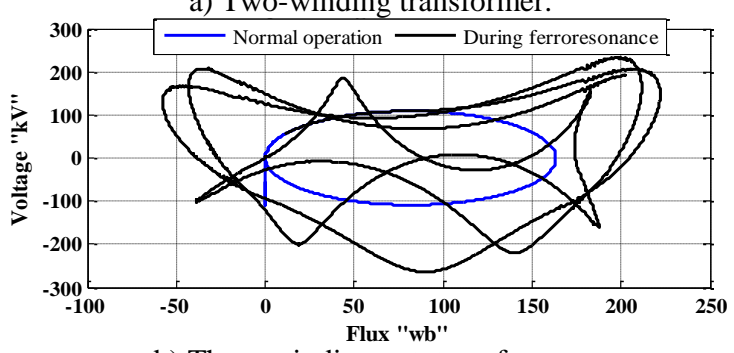

b) Three-winding autotransformer.

Figure 7- Phase-plane diagram due to ferroresonance. 
Comparing between the results shown in Figures (6) and (7), the internal transformer resonance provides sharp and fast operating loops during the transient period. The flux band corresponds to transient period are much lower than the flux band in normal operation. On the other hand, ferroresonance provides several operating loops that are distorted with higher magnitude of core flux. Consequently, the core flux is extremely dissimilar for studied cases of internal transformer resonance and ferroresonance. Therefore, flux band during the disturbance can be used for the distinguishing.

Figure (8) shows a flowchart for the flux band-based algorithm where, the core flux is computed by the integration of the voltage. Setting for voltage and flux band is selected based on the amplitude during normal operation. If an overvoltage case is detected, then if the computed flux band is higher than the setting value, ferroresonance case is detected. While, if the computed flux band is extremely lower than the setting, resonance case is detected.

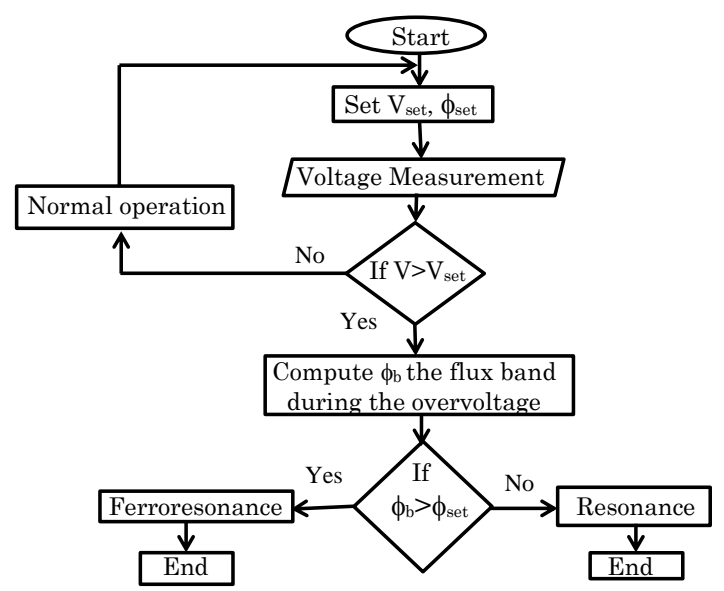

Figure 8- Flowchart of distinguishing using core flux.

\subsection{Investigation Based on Discrete Wavelet Transform (DWT)}

In this section, features of the resonance and ferroresonance overvoltages extracted using Discrete Wavelet Transform (DWT). It additionally gives an indication for the time domain analysis that is not provided by FFT or phase-plane. Daubechies Wavelet (db-4) is used as a mother wavelet. It is expected that the resonance and ferroresonance are at high and low frequencies, respectively. For attaining a single capturing sampling rate, the lowest rate can be easily attained from the high sampling rate using the down sampling technique, in which the down sampling is achieved concerning different details.

For the resonance case of the two-winding power transformer, Figure (9.a) shows the high frequency components of the wavelet decomposition in six details taken at sampling frequency $2 \mathrm{MHz}$. This sampling frequency is chosen in order to cover all possible resonance frequencies depending on the resonance frequency of the two-winding transformer. The maximum energy amplitude is shown in detail D5, which corresponds to frequency band 31.25-62.5 $\mathrm{kHz}$. This frequency band contains the high resonant frequencies observed using FFT. The low frequencies are analyzed using sampling frequency $2 \mathrm{kHz}$ as in Figure (9.b). The maximum magnitude is at the detail D5 that reflects the fundamental frequency corresponding to the steady-state overvoltages after the transient period.

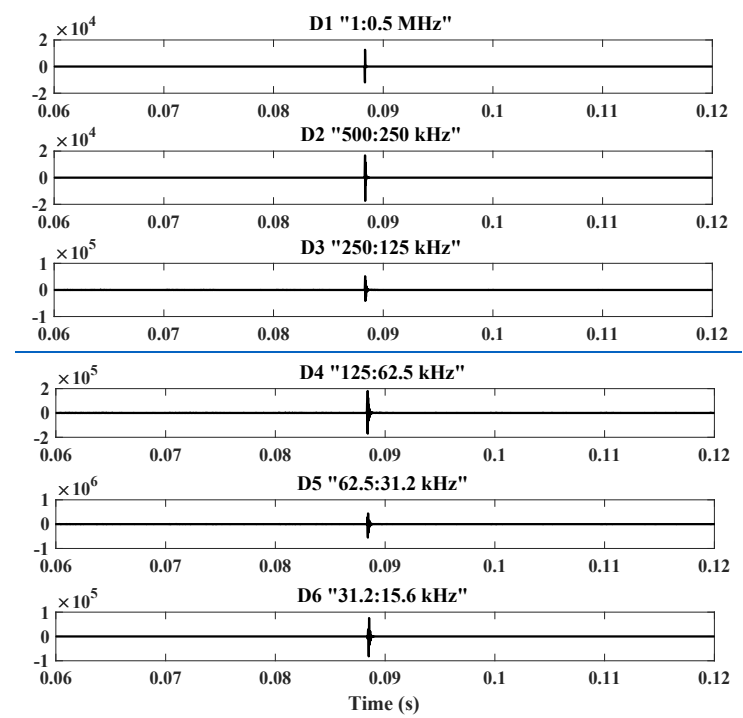

a) Sampling frequency $2 \mathrm{MHz}$.
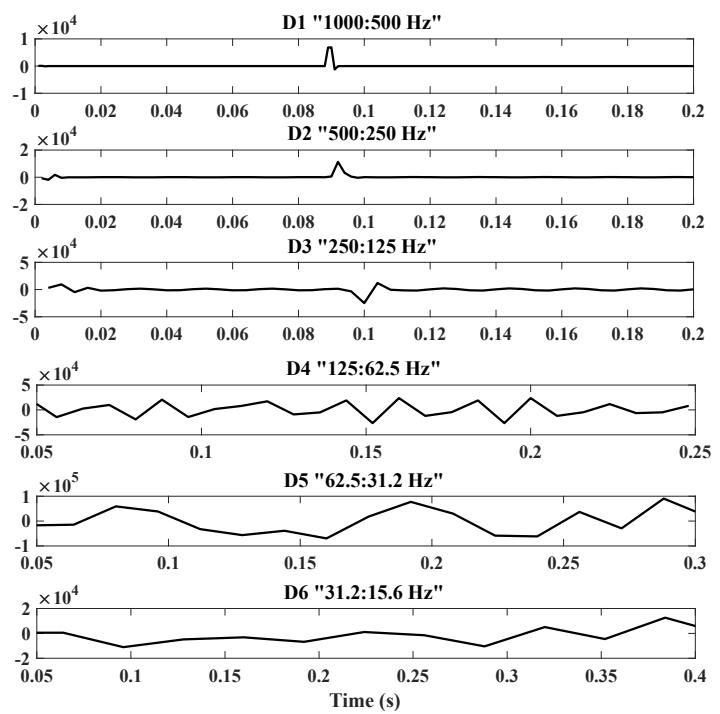

b) Sampling frequency $2 \mathrm{kHz}$.

Figure 9- DWT analyses of two-winding transformer resonance case. 
In the case of the internal resonance of the threewinding autotransformer, the high sampling rate is considered $0.2 \mathrm{MHz}$ for the transformer internal resonance evaluation, while the low sampling rate is $2 \mathrm{kHz}$ for the ferroresonance investigation. The details during internal resonance are shown in Figure (10.a), where the maximum amplitude is in detail D5 that contains the transformer resonance frequency. Figure (10.b) shows the low sampling rate details that the maximum magnitude is in detail D5 where the fundamental frequency is localized. The results in Figures (9) and (10) show a single spike for the internal resonance, where it is localized in the extracted details using a high sampling rate.

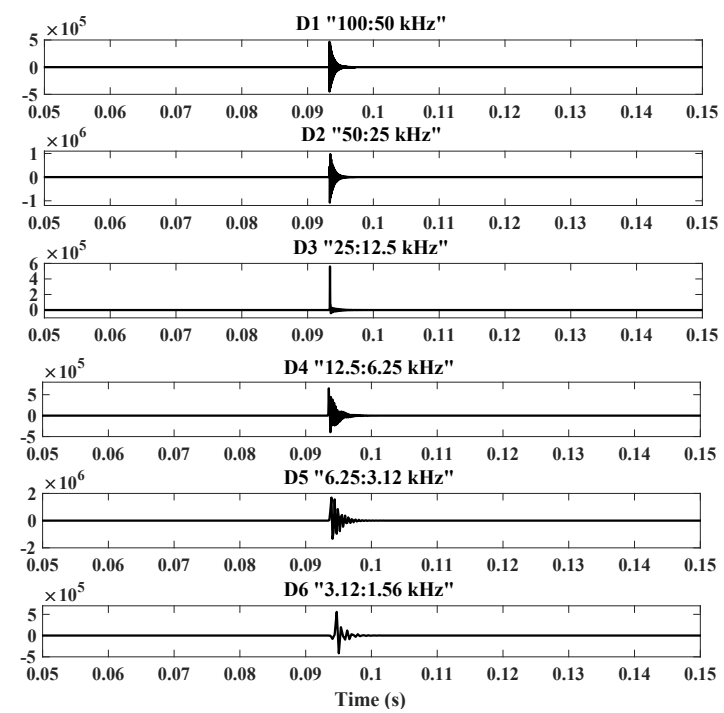

a. Sampling frequency $0.2 \mathrm{MHz}$.
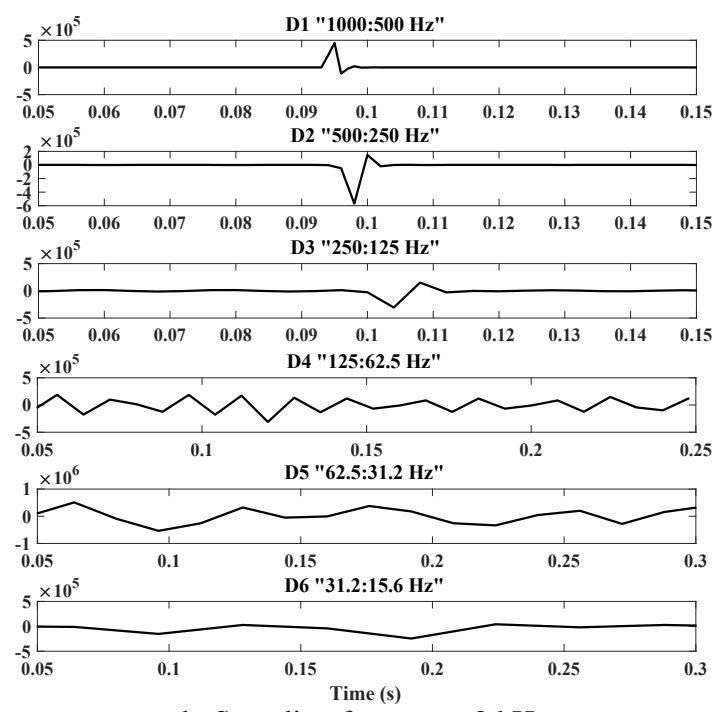

b. Sampling frequency $2 \mathrm{kHz}$.

Figure 10- DWT analyses of the autotransformer internal resonance.
The resulted ferroresonance overvoltages are also analyzed using the same procedure, and the corresponding results are shown in Figures (11) and (12) for the two-winding power transformer and three-winding autotransformer; respectively. Unlike the resonance case, the six details for the highfrequency decomposition show several and repeated spikes during the transformer ferroresonance interaction as in Figures (11.a) and (12.a). Every spike localizes the moment of saturation transition per half cycle. However, the magnitudes for these details are much lower than the high-frequency details of the internal resonance event (depicted in Figures (9.a) and (10.a)). Figure (11.b) shows the decomposition of the two-winding transformer using a low sampling rate. The maximum amplitudes are observed in detail D5 followed by the detail D4, in which they correspond to the fundamental frequency and the second harmonic, respectively. This feature confirms the saturation due to ferroresonance. Also, in case of the three-winding autotransformer depicted in Figure (12.b), the maximum amplitudes are in details D6, D5, and D4 that contain the subharmonics, fundamental frequency, and second harmonic, respectively. Also, these results show that the magnitudes for the low frequencies in the case of ferroresonance are higher than in the case of resonance, that is consistent with the FFT analysis.

There are two salient features found from DWTbased processing and comparison of the resonance and ferroresonance waveforms. The first feature is attained for the ferroresonance event that the localized repetition of transients during the halfcycles due to magnetizing transitions. This feature is salient for ferroresonance and not for the resonance. The second feature is by comparing details extraction at a high sampling rate with that at a low sampling rate. The DWT details values at high sampling rate are higher than the output at a lower sampling rate that is for resonance case and vice versa is for the ferroresonance case. Accordingly, adaptive resonance and ferroresonance detection can be obtained using these two features consequently as follows:

1. Extract the DWT details for high-frequency decomposition and low-frequency one. If the high-frequency details have one spike at the instant of the overvoltage, then the resonance case is identified as can be concluded from the results shown in Figures (9) and (10). Further security is considered that the amplitude of details using a high sampling rate is higher than the amplitude considering the low sampling rate. 


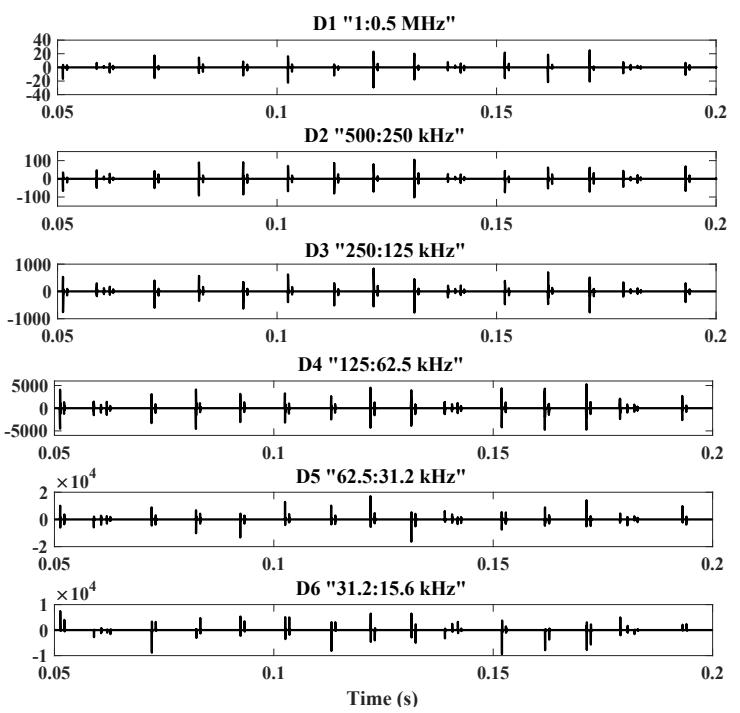

a. $\mathrm{Fs}=2 \mathrm{MHz}$.
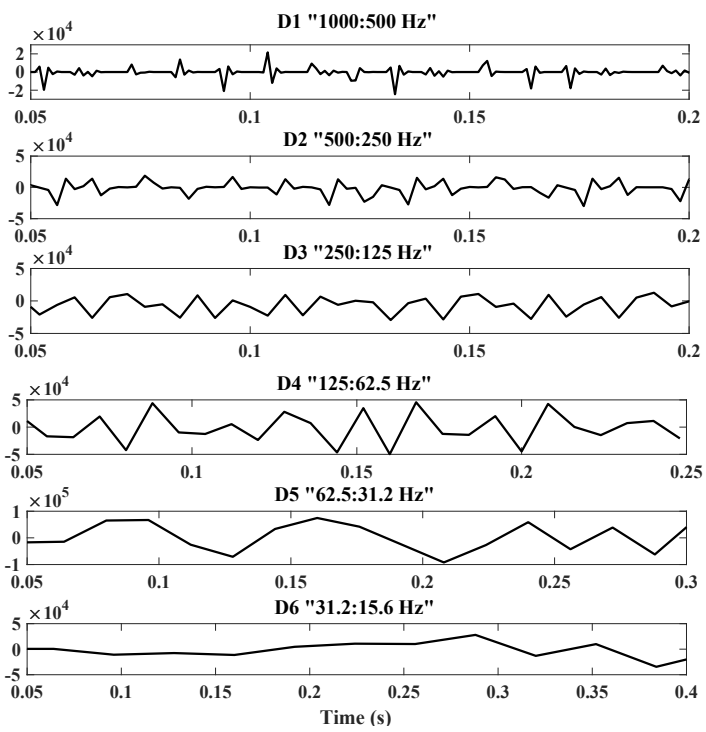

b. $\mathrm{Fs}=2 \mathrm{kHz}$.

Figure 11- DWT analyses of the two-winding transformer ferroresonance test case.

2. If the high-frequency details show repeated spikes that represent the repetition of saturation transitions, then the ferroresonance case is detected as in Figures. (11) and (12). A further feature is that the amplitudes of the lowfrequency details are higher than the amplitude of the high-frequency details.

The above distinguishing procedure can be implemented using the steps of the flowchart shown in Figure (13).
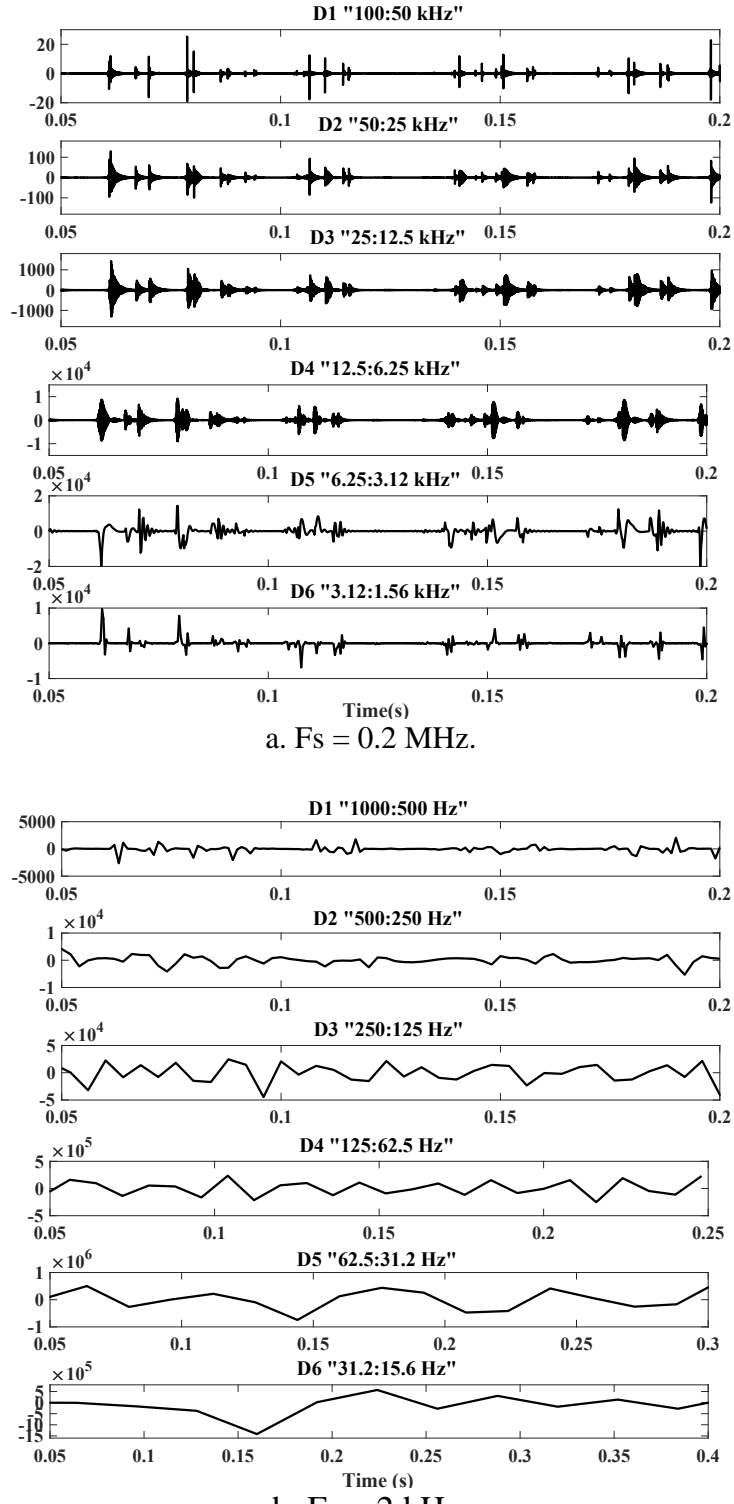

b. Fs $=2 \mathrm{kHz}$.

Figure 12- DWT analysis of the autotransformer ferroresonance test case.

\subsection{Algorithms Integration}

The above sections show three algorithms for the detection and distinguishing between internal resonance and ferroresonance overvoltages measured at the transformers secondary side. Each of these overvoltages has its salient features that can be recognized by any of these algorithms. FFT can give an indication for the resonance frequencies either high frequency during internal resonance or low frequencies during ferroresonance. However, the FFT does not give any information about the time-domain performance. 


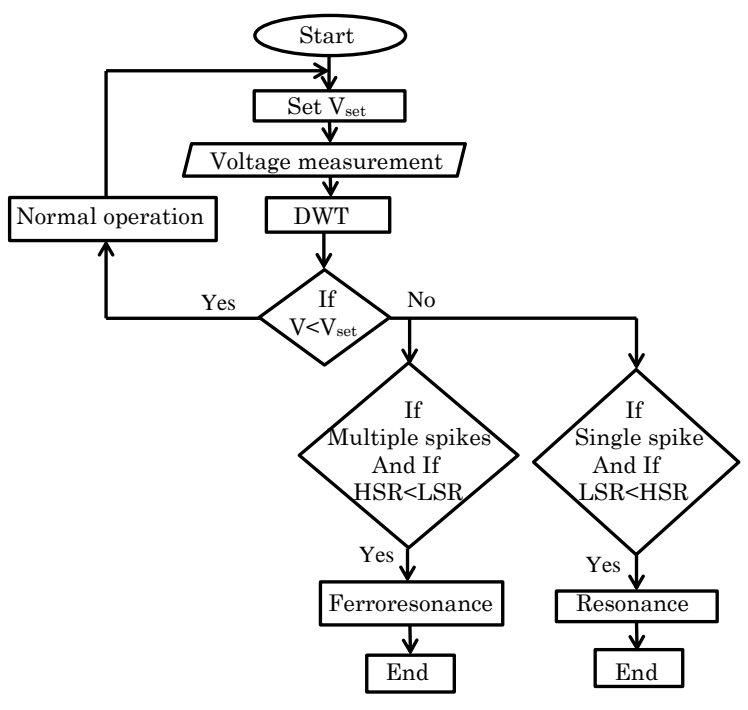

Figure 13- DWT-based distinguishing flowchart.

Features extracted using the phase-plane diagram during ferroresonance may be overlapped with other transients like load rejection case. Therefore, integration between these algorithms can overcome the shortage of each individual one. For example, the internal transformer resonance can be securely detected and distinguished by the integration between FFT and phase-plane diagram because the FFT defines the high frequencies during internal resonance and the phase-plane diagram confirms these high frequencies as it shows the extremely low flux bands during the transient period. Furthermore, the DWT shows a single spike and higher details amplitude at a high sampling rate than the details amplitude at a low sampling rate. While in the case of ferroresonance, the ferroresonance mode and its frequency can be detected by FFT, but it may be overlapped with the low frequencies during load rejection or power swing. The repeated spikes extracted using DWT can distinguish the ferroresonance interaction as it confirms the saturation transitions interaction repetitions. A further new feature is found within the DWT details that the details amplitudes of the high sampling rate are relatively lower than the details extracted at the low sampling for the same ferroresonance case. Generally, combining these algorithms together provides a reliable detecting approach as the transient features are extracted using different methods.

\section{Conclusions}

Three concepts have been discussed in order to detect and distinguish between the transformer internal resonance and ferroresonance cases using FFT, phase-plane diagram, and DWT. The core flux and the transient overvoltages at the transformer secondary side have been used as the inputs for these algorithms. The features extracted by each algorithm gave an indication for resonance or ferroresonance occurrence. The phase-plane diagram showed the effect of each case on both voltage and core flux. An adaptive and novel detector for each phenomenon has been obtained using the DWT algorithm. The recommendation has been to integrate these algorithms for reliably detecting the overvoltage phenomenon. In work to follow, incorporating these algorithms into condition monitoring or protective devices is to be evaluated in order to save the power transformers.

\section{References}

[1] B. Gustavsen "Study of Transformer Resonant Overvoltages Caused by Cable-Transformer High-Frequency Interaction" IEEE Transactions on Power Delivery, Vol. 25, No. 2, April 2010, pp. $770-779$.

[2] J. A. Halladay and C. H. Shih "Resonant Overvoltage Phenomena Caused by Transmission Line Faults" IEEE Transactions on Power Apparatus and Systems, Vol. PAS104, No. 9, Sep. 1985, pp. 2531-2539.

[3] S. Pramanik, S. Anees, and L. Satish "Interleaved Winding and Suppression of Natural Frequencies" IET Electric Power Application, Vol. 7, No. 4, 2013, pp. 237-244.

[4] M. Florkowski, J. Furgal and P. Pajak "Analysis of Fast Transient Voltage Distributions in Transformer Windings under Different Insulation Conditions" IEEE Transactions on Dielectrics and Electrical Insulation, Vol. 19, No. 6, Dec. 2012, pp. 1991-1998.

[5] P. Mitra, A. De and A. Chakrabarti "Resonant Behavior of EHV Transformer Windings under System Originated Oscillatory Transient Overvoltages" Journal on Electrical Power and Energy Systems, Vol. 33, No. 10, Dec. 2011, pp. $1760-1766$.

[6] A. De, D. Debhnath and A. Chakrabarti "A Study of the Impact of Low-Amplitude Oscillatory Switching Transients on Grid Connected EHV Transformer Winding in a Longitudinal power Supply" IEEE Transactions on Power Delivery, Vol. 24, No. 2, April 2009, pp. 679-686. 
[7] P. Mitra, A. De and A. Chakrabarti "Response of EHV Transformers to Systems-Originated Oscillatory Switching Transients" IEEE Transactions on Power Delivery, Vol. 27, No. 1, Jan. 2012, pp. 224-235.

[8] A.H. Soloot, H.K. Høidalen, and B. Gustavsen "Modeling of Wind Turbine Transformers for the Analysis of Resonant Overvoltages" Journal on Electric Power Systems Research, 2014, pp. $1-9$.

[9] "Ferroresonance" Technical Bulletin 004a May 29, 2002. [Online].

http://www.cadickcorp.com.

[10] P. Ferracci "Ferroresonance" Groupe Schneider: Cahier technique no 190, March 1998, pp. 1-28. [Online]. http://www.schneiderelectric.com.

[11] K. Milicevic, D. Vinko, and D. Vulin "Experimental Investigation of Impact of Remnant Flux on the Ferroresonance Initiation" Journal on Electrical Power and Energy Systems, Vol. 61, Oct. 2014, pp. 346-354.

[12] P. S. Moses, M. A. Masoum, and H. A. Toliyat "Impacts of Hysteresis and Magnetic Couplings on the Stability Domain of Ferroresonance in Asymmetric Three-Phase Three-Leg Transformers" IEEE Transactions on Energy Conversion, Vol. 26, No. 2, June 2011, pp. 581592.

[13] P. S. Moses and M. S. Masoum, "Modeling Ferroresonance in Asymmetric Three-Phase Power Transformers" Power Engineering Conference, (AUPEC), Adelaide, SA, Sep. 2730, 2009, pp. 1-6.

[14] A. Rezaei-Zare and M. Sanaye-Pasan "Analysis of Ferroresonance Modes in Power Transformers Using Preisach-Type Hysteretic Magnetizing Inductance" IEEE Transactions on Power Delivery, Vol. 22, No. 2, April 2007, pp. 919-929.

[15] B. A. Mork and D. L. Stuehm "Application of Nonlinear Dynamics and Chaos to Ferroresonance in Distribution Systems" IEEE Transactions on Power Delivery, Vol. 9, No. 2, April 1994, pp. 1009-1017.

[16] R. A. Walling "Ferroresonance in Low-Loss Distribution Transformers" Power Engineering
Society General Meeting, (PESGM), IEEE, Vol.2, July 13-17, 2003.

[17] L. B. Viena, F. A. Moreira, N. R. Ferreira and N. C. de Jesus "A Comparative Analysis of Transformer Models Available in the ATP Program for the Simulation of Ferroresonance" Int. Conf. on Power Systems Transients, (IPST), Delft, the Netherlands, June 14-17, 2011.

[18] M. S. ElNozahy, R. A. El-Shatshat, and M. M. Salama "A Robust Technique for Overvoltages Classification in Power Transformers" Power and Energy Society General Meeting, (PESGM), IEEE, San Diego, CA, July 22-26, 2012, pp. 1-8.

[19] G. Mokryani, M. R-. Haghifam, and J. Esmaeilpoor "Identification of Ferroresonance Based On Wavelet Transform and Artificial Neural Networks" Power Engineering Conference, (AUPEC), Perth, WA, December 9-12, 2007, pp. 1-6.

[20] Z. Bo and L. Tiecheng "On the Use of Wavelet Decomposition for Ferroresonance Detection in Power System" Int. Conference on Power and Energy Engineering, (APPEEC), Wuhan, March 27-31, 2009, pp. 1-4.

[21] M. A, Beg, M. K. Khedkar, and G. M. Dhole "Discrimination of Capacitor Switching Transients using Wavelet" Journal of science, spirituality, business and technology, Vol. 1, No.1, March 2012, pp. 34-37.

[22] J. Izykowski, E. Rosolowski, and K. Fatla "Discrimination between Internal Fault and Magnetizing Inrush Current in Transformers using Wavelet Transform". [Online]. http://www.academia.edu.

[23] A. H. A. Hamza, T. K. Alnemran and A. S. Abd-elshafy "Discrimination Technique between Transformer Inrush Current and Internal Fault Current using D1 Wavelet Coefficient Based ANFIS" Journal of Engineering Science and Technology, Vol.3, No.4, Aug. 2013, pp. 655-662.

[24] ATPDraw version 3.5 for Windows 9x/NT/2000/XP Users' Manual.

[25] A. A. Nassar, "Investigation of Resonance and Ferroresonance Overvoltages due to CableTransformer Interaction," M.Sc. thesis, 
Electrical Engineering. Dept., Minoufiya. University, Shebin Elkom, Egypt, Aug. 2014.

\section{Appendix}

Excitation and short circuit test data for the two transformers are summarized in Tables (1) and (2). The magnetizing characteristic for the transformers core is shown in Figure (14). The power cables are arranged in trefoil formation in the ground at $1 \mathrm{~m}$ depth and its details are in Table (3).

Table 1- Two-winding power transformer data [24].

\begin{tabular}{cc}
\hline \hline Description & ataD \\
\hline \hline Ratings and & MVA $, 132 / 15 \mathrm{kV}, 155$ \\
connection & $\mathrm{Y} / \mathrm{d} 11$ \\
Excitation current & $\mathrm{A} 2.67 / \% 0.3$ \\
Excitation losses & $\mathrm{kW} \mathrm{74}$ \\
Short circuit losses & $\mathrm{kW} \mathrm{461}$ \\
Short circuit & $\% 14$ \\
reactance & $\%$ \\
\hline \hline
\end{tabular}

Table 2- Three-winding autotransformer data [24].

\begin{tabular}{lc}
\hline \hline \multicolumn{1}{c}{ Description } & ataD \\
\hline \hline Voltage rating and connection & $\mathrm{kV}$, Yyd11 18/132/400 \\
Power rating & MVA 250 \\
+ve seq. excitation loss/current & $\% \mathrm{~kW} / 0.2140$ \\
+ve seq. reactance: HL, HT, LT & $\%$ 24 41.6667 ،15 \\
Short circuit losses: HL, HT, LT & $\mathrm{kW} \mathrm{159،188،710}$ \\
\hline \hline HL: High to Low, HT: High to Tertiary, LT: Low to Tertiary.
\end{tabular}

Table3-.Cables data

\begin{tabular}{lcc}
\hline \multicolumn{1}{c}{ Description } & $\mathrm{kV} \mathrm{400}$ & $\mathrm{kV} \mathrm{120}$ \\
& Cable & Cable \\
\hline \hline Rated voltage for the power cable & $\mathrm{kV} \mathrm{400}$ & $\mathrm{kV} \mathrm{120}$ \\
Cross section of conductor $\left(\mathrm{mm}^{2}\right)$ & 2500 & 1200 \\
Diameter of conductor $(\mathrm{mm})$ & 62 & 42.8 \\
Insulation thickness $(\mathrm{m})$ & 27 & 13 \\
Diameter over insulation $(\mathrm{mm})$ & 121 & 73.8 \\
Outer diameter of cable $(\mathrm{mm})$ & 140.8 & 89.5 \\
Conductor resistivity $(\mathrm{m} \Omega . \mathrm{mm})$ & 0.023 & 0.023 \\
Ground resistivity $(\Omega)$ & 20 & 20 \\
Relative permeability of the conductor & 1 & 1 \\
Relative permeability of the insulation & 1 & 1 \\
Relative permittivity of the insulation & 2.7 & 2.7 \\
\hline \hline
\end{tabular}

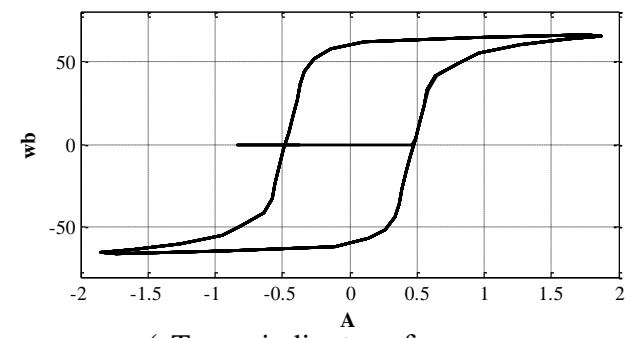

(aTwo-windingtransformer .

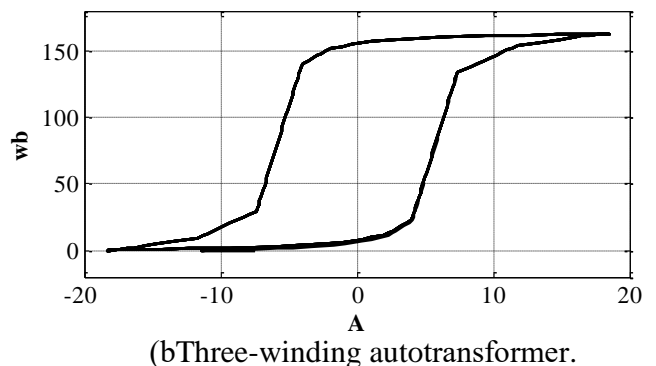

Figure 14 -Taken hysteresis characteristics for the simulatedpower .transformers 